\title{
Scene Generation and Target Detection Development for HWIL Simulation
}

\author{
Ryan E. Sherrill* \\ Auburn University, Auburn, AL, 36849, USA
}

\begin{abstract}
HWIL simulations are useful tools in developing and testing complex systems at lower cost than experimental tests. In order to model a missile intercept, the missile's optical sensor must be stimulated by an artificial environment that accurately represents the physical world. This paper describes the methods used for developing scene-generation and target-detection computer subroutines in the HWIL lab at Auburn University.
\end{abstract}

\section{Introduction}

The purpose of a Hardware-in-the-Loop (HWIL) simulation is to model as accurately as possible the response of physical components to a simulated environment. This technology has multiple uses for military missiles including: the testing of new systems and components; quality control for manufacturing processes; and reliability for stockpiled components. In HWIL testing, the missile is mounted to a table or a flight motion table, facing a projected scene. The missile's seeker responds to the scene as if in a real flight, passing information and directing responses from the rest of the missile's systems. ${ }^{4}$ Numerous simulated engagements can be presented to each missile and its performance evaluated, offering a wider range of data than would be possible in a live-fire test, at a much lower cost.

With collaboration from the US Army, Auburn University is developing a HWIL facility for educational and research purposes. Once operational, it will provide training for students on complex simulations, model development, and the testing of unclassified hardware. As part of this effort, a computer simulation was developed previously that models accurately the flight of a missile in six dimensions as it intercepts a ballistic target. One goal of the current project was to increase the simulation capability by augmenting the system with a scene generation program. Another goal was to develop a target detection program that simulates a missile seeker. This allows computer software to be used in the HWIL facility, replacing hardware components that have limited availability.

\section{System Development}

\section{A. Scene Generation}

The engagement scenario that was modeled is an intercept in the upper atmosphere. The camera position and orientation control the scene observed by the seeker. The camera field of view (FOV) and resolution can also be adjusted to model various seekers. Initial tests were performed with a FOV of 10 degrees and pixel resolution of $n_{x}=n_{y}=500$. MATLAB version 2007a was used for this project. Using MATLAB's rendering tools, the scene is created against a black background. Next, a field of stars is added to the generated scene. This models accurately the intercept conditions, as stars appear in infrared images of the night sky. Also, the target detection program must distinguish the difference between a target and its background. The stars were modeled by uniformly distributing points on a sphere. Each point was modeled as a positive charge, and using the law of repulsion between positive charges, the points diffused themselves evenly on the sphere's surface. The resulting celestial sphere is shown in Fig. 1. The camera is located inside the sphere and looks outward; therefore, at any instant only a small section of stars can be seen, depending on the camera FOV and orientation.

\footnotetext{
${ }^{*}$ Graduate Research Assistant, Department of Aerospace Engineering, 211 Davis Hall, AIAA Student Member.
} 


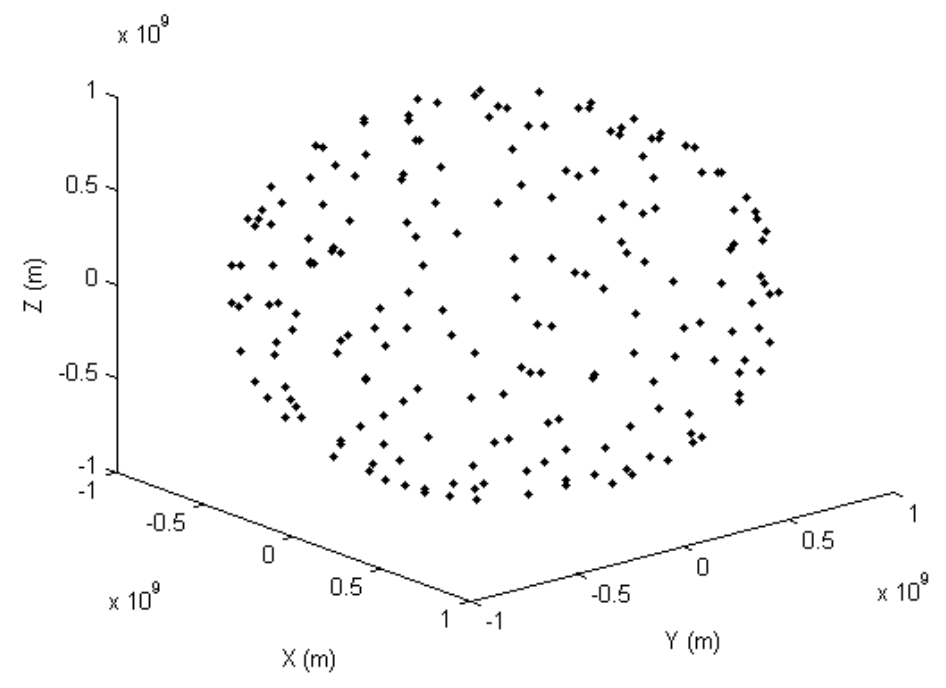

Figure 1. The spherical star field used to represent the night sky.

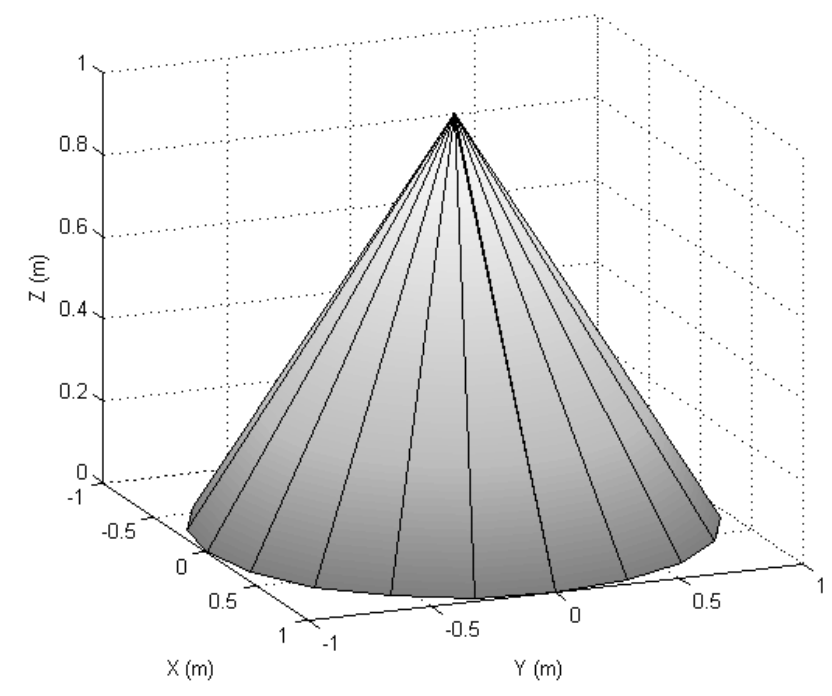

Figure 2. Visual target model.

The target is the final portion of the image to be generated. A ballistic warhead was modeled as a cone two meters tall by one meter in diameter, as shown in Fig. 2. Infrared sensors only can see the intensity of light at a given wavelength. Most military interceptor seekers examine only the infrared portion of light between 0.4 and $0.7 \mu \mathrm{m}$. Objects entering the Earth's atmosphere typically experience temperatures around 900 to 1200 degrees Celsius; ${ }^{3}$ therefore they strongly emit in the specified band, making them easier to discriminate against the atmosphere. The intensity of the cone was varied from white at the tip to gray at the base to model the temperature distribution that would be seen by an infrared sensor. The stars were given the same intensity value as the base of the target. Fig. 3 shows the complete image from the scene generation program.

In HWIL testing, the resulting scene can be projected to stimulate the seeker under test. Depending on the operational wavelength of the sensor, this can be done with an infrared projector or a more conventional monitor. The following subsection describes a target detection algorithm that can be used in digital simulation by capturing directly the rendered scene. 


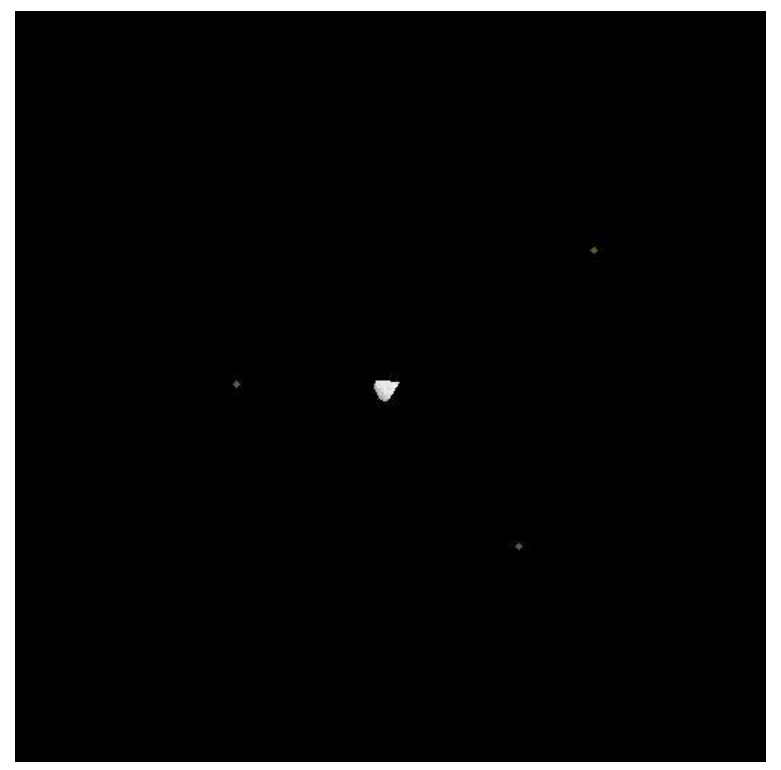

Figure 3. The computer generated target used for intercept simulations.

\section{B. Target Detection}

A target-detection module was created to simulate the behavior of a missile seeker. It was assumed that the engagement scenario involved a single interceptor and target, and that the target could not deploy decoys or other countermeasures. Therefore, the target detection searches the captured image for the value of the highest intensity pixel, and locates all pixels with that intensity value. A baseline threshold was initially set well below the intensity of the target and stars, but above the level of the image background. The target was located by analyzing each pixel in a two-dimensional rectilinear pattern, also called a Raster scan, ${ }^{1}$ as shown in Fig. 4. The intensity of all pixels were compared to find the highest intensity. All pixels with that intensity were identified as the target and their pixel locations $\left(x_{i}, y_{i}\right)$ were extracted. The Raster scan of a 500 pixel by 500 pixel image is computationally expensive, greatly slowing down the simulation. Therefore, a modified procedure was implemented after the initial image detection, as shown in Fig. 5. Once the target is detected, the seeker is steered to keep the target in the center of the field of view. Therefore, the target will ideally be located near the center of the image. Using this fact, the 100 pixels in the middle of the image are initially scanned. If an object is detected that has the same intensity value as the initial scan, it is assumed that the target has been located and the azimuth and elevation angles in addition to the line-of-sight (LOS) are calculated as described above. If no object is found, the entire image is analyzed to re-locate the target. This method reduces the search time by approximately two orders of magnitude.

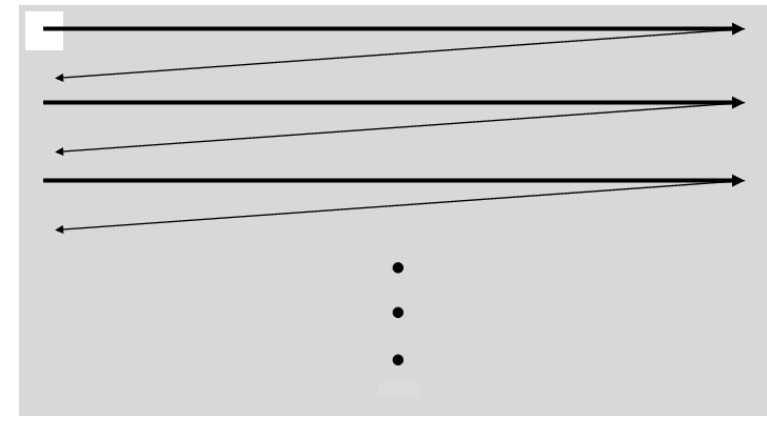

Figure 4. Raster scan pattern. 


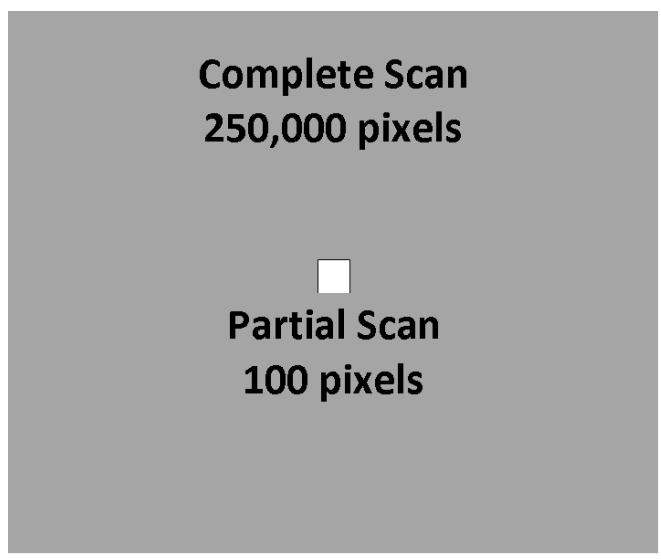

Figure 5. Reduction of scan area.

The centroid of the target is determined by Eq. (1).

$$
\begin{aligned}
& \bar{X}=\frac{1}{n} \sum_{i=1}^{n} x_{i} \\
& \bar{Y}=\frac{1}{n} \sum_{i=1}^{n} y_{i}
\end{aligned}
$$

Here $n$ is the number of pixels in the target image. Using the target centroid, the line of sight to the target can be calculated. This is done using the pin-hole camera model shown in Fig. 6. Point $O$ represents the seeker aperture. The camera parameters $h, w$, and $f$ represent the image height, width, and focal length. The position of the target relative to the camera is given by the vector $\mathbf{P}$. Although range to the target can not be determined from the image, the position of the target image, $\hat{\mathbf{P}}$, can be calculated. Here, the camera parameters $\frac{w}{f}$ and $\frac{h}{f}$ can be calculated from the FOV specifications.

$$
\begin{aligned}
& \frac{w}{f}=2 \tan \left(F O V_{x}\right) \\
& \frac{h}{f}=2 \tan \left(F O V_{y}\right)
\end{aligned}
$$

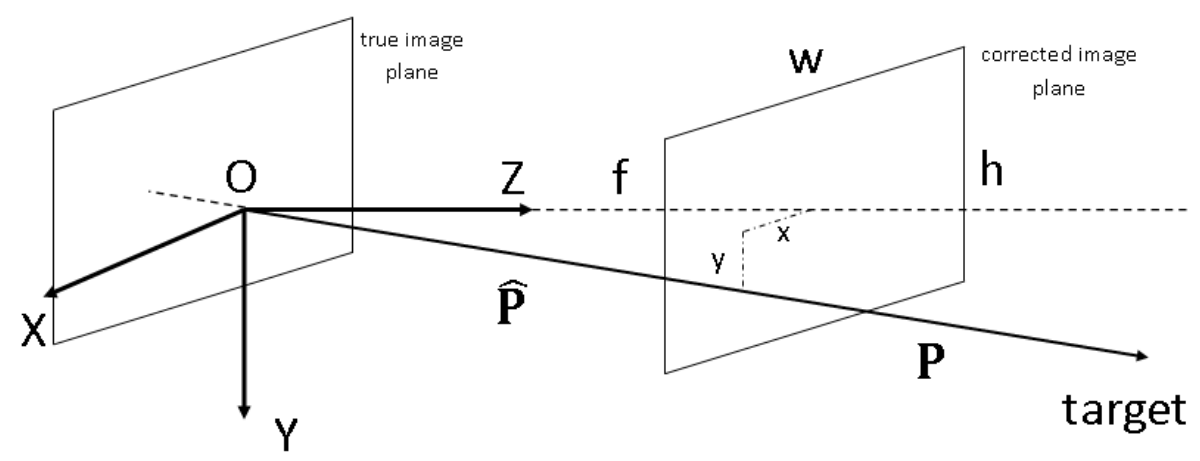

Figure 6. Pinhole camera model. The angle from the camera to the focal plane is the same as the angle from the camera to the target 


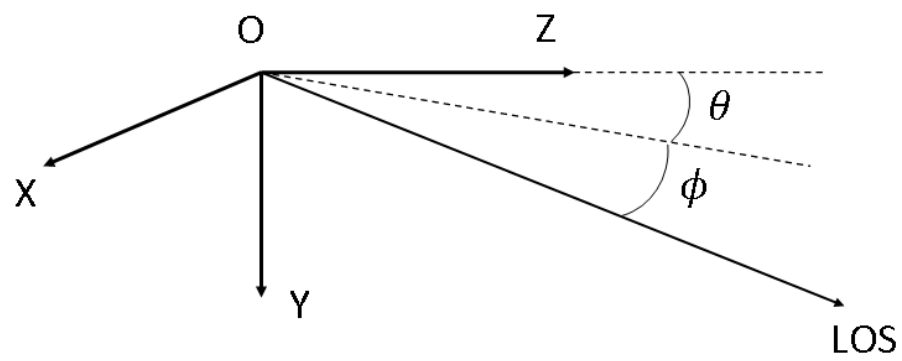

Figure 7. Comparing azimuth and elevation angles to the LOS vector.

The percentage from the center of the image that the target is located can then be calculated.

$$
\begin{aligned}
& \frac{x}{f}=\left(\frac{2 \bar{X}-n_{x}}{n_{x}}\right)\left(\frac{w}{f}\right) \\
& \frac{y}{f}=\left(\frac{2 \bar{Y}-n_{y}}{n_{y}}\right)\left(\frac{h}{f}\right)
\end{aligned}
$$

The LOS vector can be constructed as shown.

$$
L O S_{\text {camera }}=\frac{1}{\sqrt{x^{2}+y^{2}+f^{2}}}\left[\begin{array}{l}
x \\
y \\
f
\end{array}\right]=\frac{1}{\sqrt{\left(\frac{x}{f}\right)^{2}+\left(\frac{y}{f}\right)^{2}+1}}\left[\begin{array}{c}
\frac{x}{f} \\
\frac{y}{f} \\
1
\end{array}\right]
$$

The missile guidance system requires the LOS from the missile to the target in inertial coordinates. Using a previously calculated direction cosine matrix from the guidance system, the LOS vector in Eq. (4) is converted into inertial coordinates to steer the missile. The azimuth and elevation angles shown in Fig. 7 are required to steer the missile's seeker. Using Eq. (5), the LOS vector is covered to these two angles.

$$
\begin{aligned}
\phi & =\sin ^{-1}\left(\frac{\frac{y}{f}}{\left(\frac{x}{f}\right)^{2}+\left(\frac{y}{f}\right)^{2}+1}\right) \\
\theta & =\tan ^{-1}\left(\frac{x}{f}\right)
\end{aligned}
$$

\section{Results}

A series of computer experiments were conducted using Software-in-the-Loop simulations. All experiments were conducted on a personal computer with a $2.2 \mathrm{GHz}$ processor with simulation run times of about 5 minutes. The results demonstrate that the target detection program could correctly identify the target at every time step. Four different engagement scenarios were tested, with the target following a constant velocity parabolic path, a horizontal sinusoidal path, a vertical sinusoidal path, and finally a helical path. The deviations allowed for the sinusoidal and helical paths were $1 \mathrm{~km}$ from a pure ballistic trajectory. Figures 8-12 show the error angle in radians between the calculated LOS and the true LOS for the interceptor's flight. Only the final 0.5 seconds are displayed because the error rate was negligible until that point. Although the errors remain minor, they increase near the final time as the target's apparent diameter increases with decreasing range. 


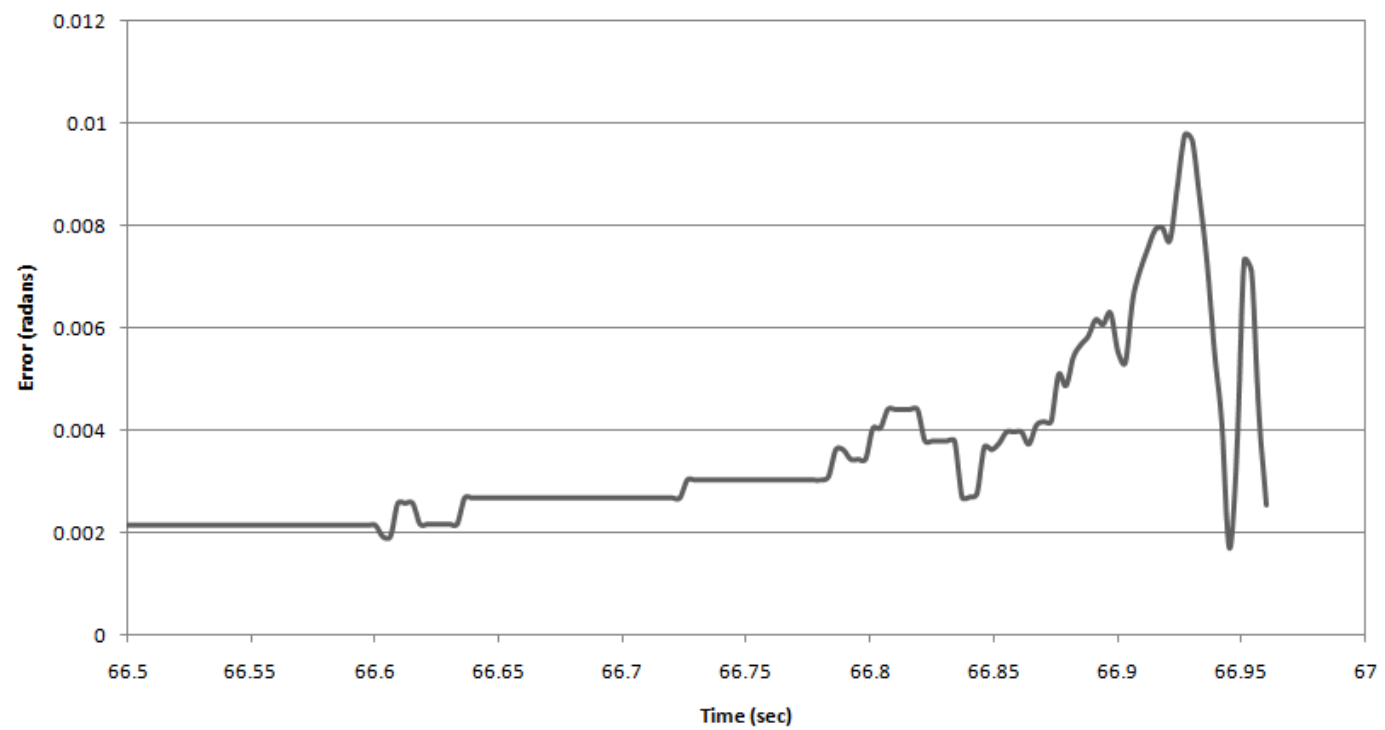

Figure 8. Error in LOS during the final 0.5 seconds of simulation for a constant velocity parabolic target trajectory.

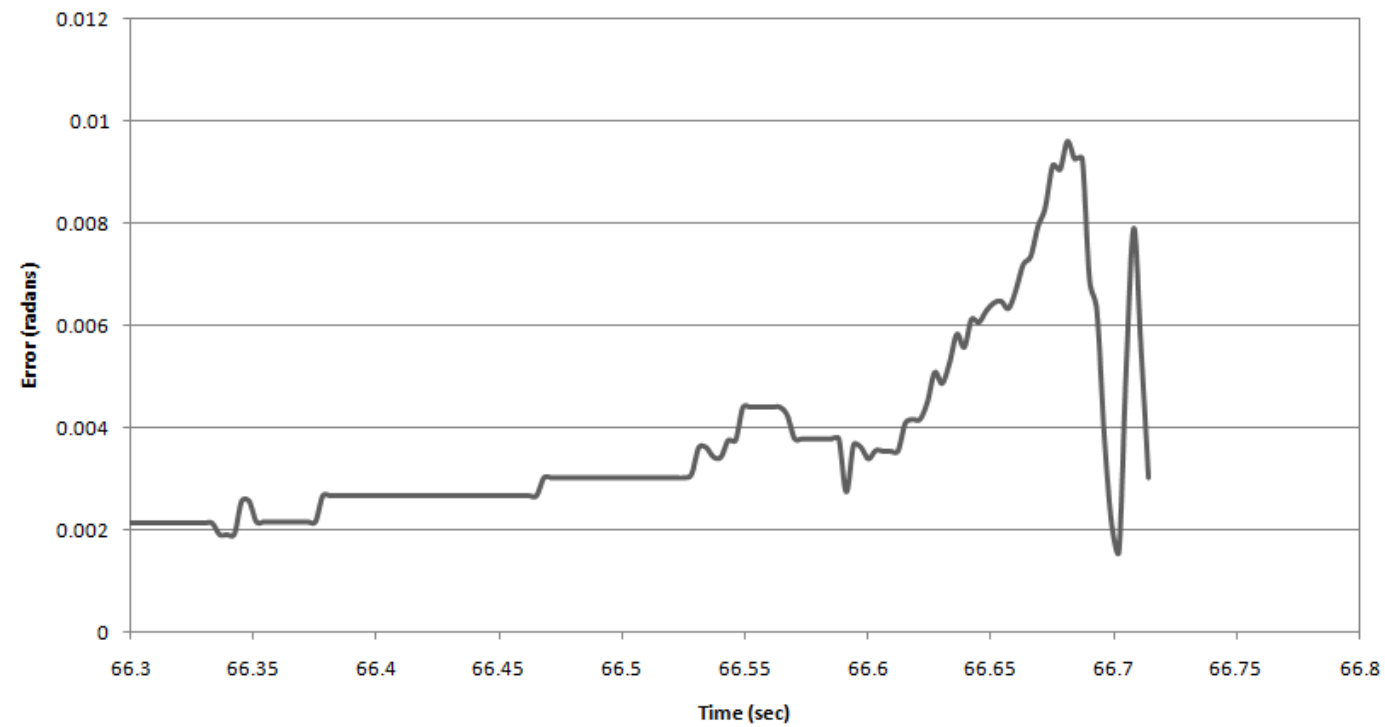

Figure 9. Error in LOS during the final 0.5 seconds of simulation for a target trajectory with horizontal sinusoidal deviations. 


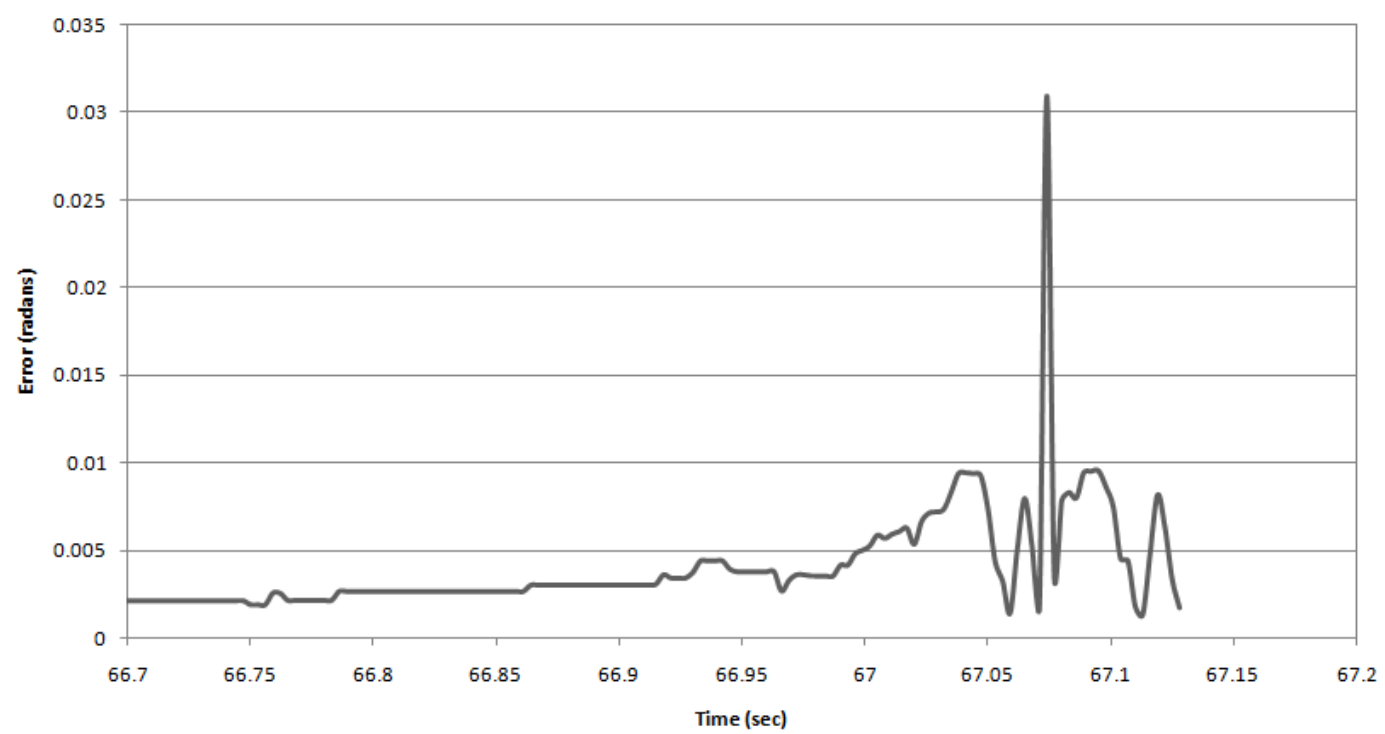

Figure 10. Error in LOS during the final 0.5 seconds of simulation for a target trajectory with vertical sinusoidal deviations.

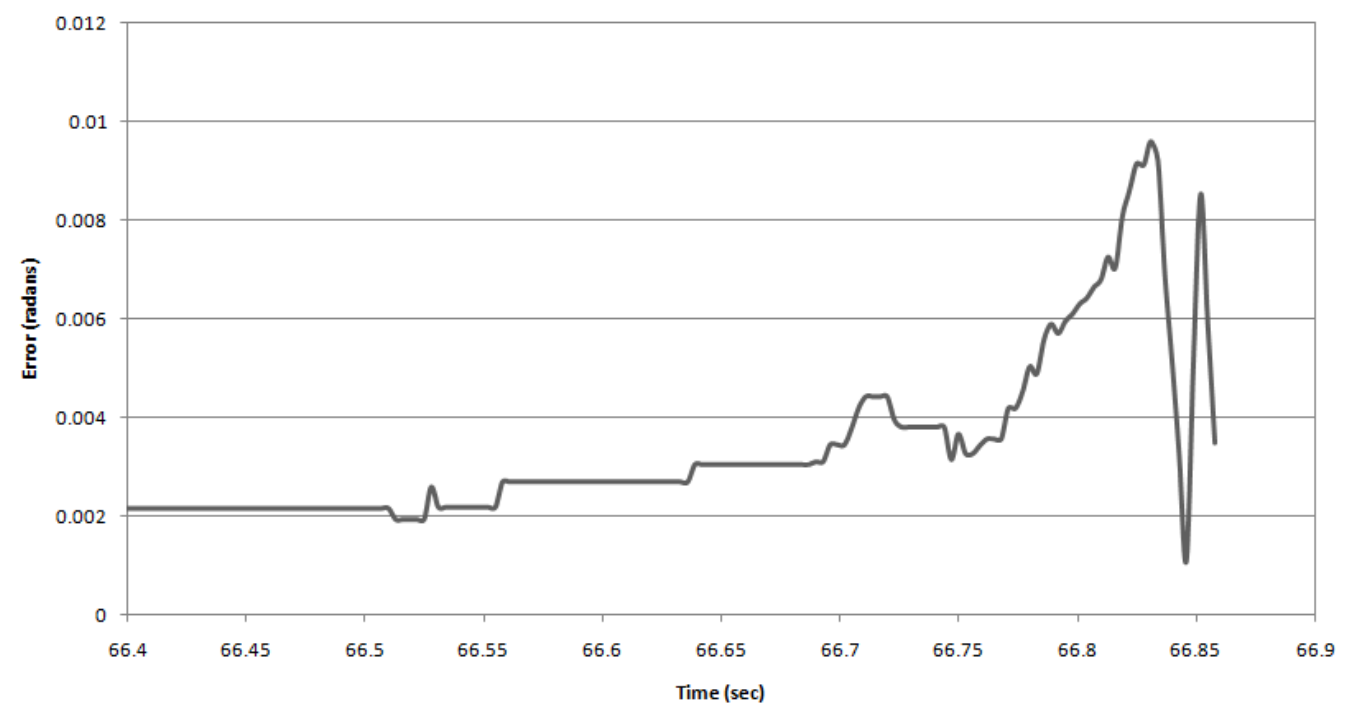

Figure 11. Error in LOS during the final 0.5 seconds of simulation for a helical target trajectory. 


\section{Conclusion}

The ultimate goal in the HWIL laboratory is to develop distributed simulation that can be used for missile testing. The results presented here show the ability to generate an image that simulates accurately a missile intercept scenario. Using a target-detection simulation of a missile seeker, the target is located at every stage of flight with minor LOS error. Future efforts will focus on adapting the current progress to static HWIL simulations as a building block to dynamic HWIL testing.

\section{Acknowledgments}

The author would like to thank three Auburn University professors for their assistance during this research project. His principal advisor, Dr. Andrew Sinclair, provided continued support and patience throughout this process. His help was essential in developing the MATLAB subroutines described above. Dr. Brian Thurow's knowledge of optical devices proved invaluable when constructing the simulation elements. Finally, the author is grateful to Dr. John Cochran Jr. for having faith in the author's abilities and inviting him to work on this research project.

\section{References}

${ }^{1}$ Driggers, R., Cox, P., and Edwards, T., Introduction to Infrared and Electro-Optical Systems, Artech House, Boston, 1999.

${ }^{2}$ Gaskill, J., Linear Systems, Fourier Transforms, and Optics, Wiley, New York, 1978

${ }^{3}$ Martin, J., Atmospheric Reentry; an Introduction to its Science and Engineering, Prentice-Hall, Englewood Cliffs, N.J., 1966

${ }^{4}$ United States Army Redstone Technical Test Center, http://www.rttc.army.mil/whatwedo/primary_ ser/modeling/hwil.htm 